Draft version August 23, 2018

Preprint typeset using $\mathrm{L}^{\mathrm{A}} \mathrm{T}_{\mathrm{E}} \mathrm{X}$ style emulateapj v. 5/2/11

\title{
OHMIC HEATING SUSPENDS, NOT REVERSES, THE COOLING CONTRACTION OF HOT JUPITERS
}

\author{
YANQIN WU ${ }^{1}$, YoRAM Lithwick ${ }^{2}$ \\ Draft version August 23, 2018
}

\begin{abstract}
We study the radius evolution of close-in extra-solar jupiters under Ohmic heating, a mechanism that was recently proposed to explain the large observed sizes of many of these planets. Planets are born with high entropy and they subsequently cool and contract. We focus on two cases: first, that ohmic heating commences when the planet is hot (high entropy); and second, that it commences after the planet has cooled. In the former case, we use analytical scaling and numerical experiments to confirm that Ohmic heating is capable of suspending the cooling as long as a few percent of the stellar irradiation is converted into Ohmic heating, and the planet has a surface wind that extends to pressures of $\sim 10$ bar or deeper. For these parameters, the radii at which cooling is stalled are consistent with (or larger than) the observed radii of most planets. The only two exceptions are WASP-17b and HAT-P-32b. In contrast to the high entropy case, we show that Ohmic heating cannot significantly re-inflate planets after they have already cooled. This leads us to suggest that the diversity of radii observed in hot jupiters may be partially explained by the different epochs at which they are migrated to their current locations.
\end{abstract}

\section{OBSERVED RADII OF HOT JUPITERS}

Since the discovery of the first transiting hot jupiter (Charbonneau et al. 2000), the radii of these objects have remained puzzling. While cooling theory predicts that these planets, regardless of mass, should contract to $\sim 1 R_{J}$ (Jupiter radius) at the age of a few Gyrs, the observed radii for hot jupiters range from $0.8 R_{J}$ to $2 R_{J}$. Factors such as stellar irradiation, a varying core mass, and atmospheric metal content can at best cause $\sim 10 \%-20 \%$ variations in the radius (see, e.g. Burrows et al. (2007).

There is a trend that the observed radii rise with the amount of stellar irradiation (e.g., Enoch et al. 2011 Laughlin et al. 2011, also see data in Fig. 5) though the most inflated planet discovered to date (WASP-17b, Anderson et al. 2011) is not the hottest one.

Lacking a mechanism of energy generation, isolated Jovian planets inexorably contract as they cool. The inflated hot jupiters look much younger than their real ages suggest. Some mechanism is at work either to stall their aging or to rejuvenate them from old age (see proposals by, e.g., Bodenheimer et al. 2001, Chabrier \& Baraffe 2007: Youdin \& Mitchell 2010, Batygin \& Stevenson 2010). The rejuvenation may be particularly relevant for hot jupiters, as they are believed not to have formed in situ, but to have migrated inward after they formed (perhaps well after). We consider in detail the proposal of Batygin \& Stevenson (2010) where a surface wind (see review by Showman et al. 2010) blowing across the planetary magnetic field acts as a battery that sends current to the interior and gives rise to Ohmic heating in the deeper layers. A number of other studies have also discussed Ohmic heating in the atmospheres of hot jupiters (Perna et al. 2010b, 2012: Menou 2011). Our study here

\footnotetext{
${ }^{1}$ Department of Astronomy \& Astrophysics, University of Toronto, Toronto, ON M5S 3H4, Canada

2 Department of Physics \& Astronomy, Northwestern University, Evanston, IL 60208 \& Center for Interdisciplinary Exploration and Research in Astrophysics (CIERA)
}

is similar in spirit to that of Batygin et al. (2011) (hereafter B12), where they incorporat Ohmic heating (both in the atmosphere and in deeper layers) into calculations of planet thermal evolutions. They find that Ohmic heating can explain the inflated sizes of hot jupiters, and that low mass hot jupiters can be inflated to become Roche lobe overflowing. In this work, we also find that Ohmic heating appears effective in stalling the cooling contraction, largely confirming results of B12, but we find that it is incapable of re-inflating the planets after they have contracted.

Our work builds on that of Batygin \& Stevenson (2010) but we focus on the thermodynamical aspects; we elucidate the condition for Ohmic heating to be effective; and we investigate the interior structure of planets as affected by Ohmic heating. We are particularly interested in the question of planet rejuvenation. Our work shows that Ohmic heating, being a largely surface phenomenon, is ineffective at re-inflating planets, as this requires entropy deposition in the very center of planets.

\section{STALLING CONTRACTION}

\subsection{General Criterion for Stalling Contraction}

We consider the stalling of contraction in an irradiated planet. An elucidating work in this regard is Arras \& Bildsten (2006). Our discussion here is similar in spirit to theirs.

The interior of the planet is largely convective due to the high opacity there. The resulting temperature profile is adiabatic, with an internal entropy $S$. The layer below the photosphere, however, is nearly isothermal with $T \approx T_{\mathrm{eq}}$, as a result of the strong stellar irradiation. We simplify the planet into a two-zone model, an upper isothermal envelope and an isentropic core (see Fig. 11) ${ }^{3}$ We denote the depth of the transition point as $z_{\mathrm{tr}}$. This depth determines the cooling rate of the planet.

\footnotetext{
3 This simplification may break down when, e.g., due to Ohmic heating, another adiabatic zone appears near the surface. We ignore this complication here for clarity of exposition.
} 


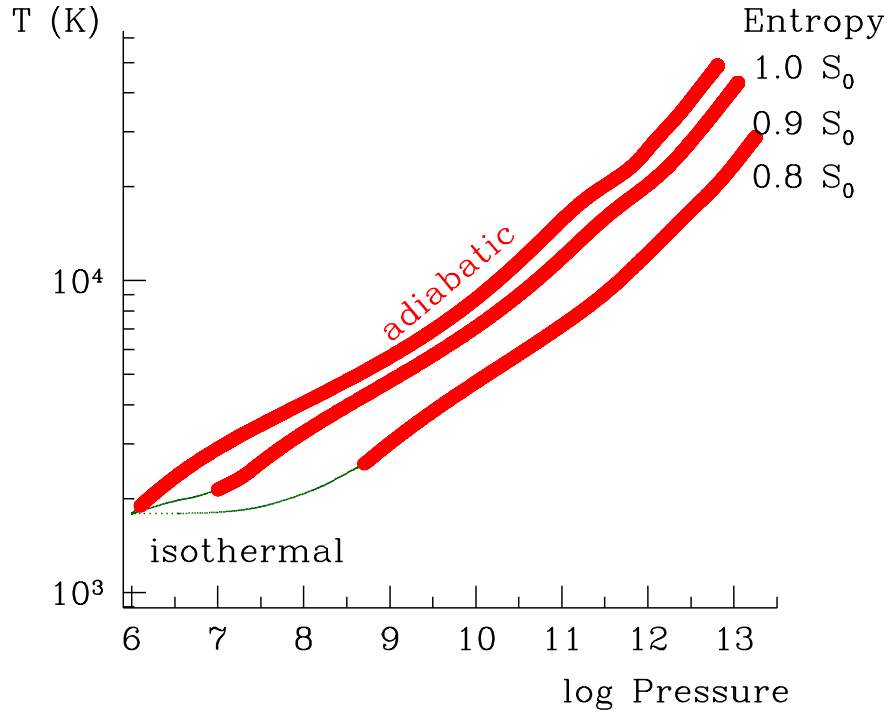

FIG. 1. - Temperature as a function of logarithmic pressure (in cgs units) inside an irradiated planet, as it cools with time. Values for the equilibrium temperature and the planet mass are chosen to resemble those of TrEs4-b: $T_{\text {eq }}=1788 \mathrm{~K}$ and $M_{p}=0.91 M_{J}$ Shown here are the three temperature profiles when internal entropy (in the isentropic core) takes values of $S=1 S_{0}, 0.9 S_{0}$ and $0.8 S_{0}$, with $S_{0}$ being value of the surface entropy. There is a oneto-one correspondence between $S$ and the planet radius (which progresses from 1.9 to 1.5 to $1.2 R_{J}$ ). Moreover, since the convection zone (marked in red) recedes as the planet cools, there is also a one-to-one correspondence between $S$ and the self luminosity (which decreases from $10^{-4}$ to $10^{-6}$ to $10^{-7} L_{\odot}$ ). These models are obtained using our thermal equilibrium code $(2.3$.

As we now demonstrate, the value of the internal entropy $(S)$ determines both the planet's radius $(R)$ and its cooling luminosity $(L)$. The first connection is straightforward - a higher $S$ corresponds to a higher internal temperature and therefore a larger scale height. The size of the planet is mostly determined by its bottommost scale height. So in a given planet, $R$ is a monotonic function of $S$.

To establish the connection between $S$ and $L$, it is useful to recognize that the planet's cooling is controlled exclusively by the optical depth and the temperature gradient at the transition point, $z_{\text {tr }}$ (Arras \& Bildsten 2006). Radiative diffusion allows an energy leakage that scales with the temperature gradient as,

$$
F_{\mathrm{rad}}=\frac{a c}{3 \kappa \rho} \frac{d T^{4}}{d z}=\frac{4 \sigma_{B}}{3} \frac{d T^{4}}{d \tau},
$$

where $\tau=\int_{0}^{z} \kappa \rho d z^{\prime}$ is the optical depth measured from the surface, and $\sigma_{B}$ the Stefan-Boltzman constant. Below $z_{\mathrm{tr}}$, the temperature gradient is adiabatic and the optical depth rises quickly; above $z_{\mathrm{tr}}$, the temperature is nearly isothermal, $T_{\mathrm{tr}} \sim T_{\text {eq }}$. The radiative flux of the entire planet is therefore set by the optical depth and the (adiabatic) temperature gradient at $z_{\mathrm{tr}}$, and is roughly $\sim \sigma_{B} T_{\text {eq }}^{4} / \tau_{\mathrm{tr}}$. And lastly, since a planet with a cooler interior has a deeper isothermal layer (larger $z_{\mathrm{tr}}$, see Fig. 1), it will therefore have a lower cooling flux.

Hence measuring the radius directly probes the internal entropy, which in turn, informs us how fast the planet is losing energy. An inflated planet has a higher entropy, and therefore has to lose heat faster. The only way to stall contraction is to supply this cooling luminosity, not from the internal energy of the planet, but from some other means (e.g., Ohmic heating, wave energy, etc.). Moreover, this extra energy source has to be able to replace the cooling flux at depth $z \geq z_{\text {tr }}$.

In our calculations, we frequently find that a new convection zone develops in the wind zone, where Ohmic dissipation is at its greatest power. This has the effect of pushing $z_{\operatorname{tr}}$ to a deeper depth, for the same interior adiabat. But it will not change the above discussion.

\subsection{Constraints on Ohmic Heating}

In this paper, we focus on the Ohmic heating mechanism (Batygin \& Stevenson 2010), which is one of the best laid-out mechanisms for halting contraction. In this model, if the planet has a large scale magnetic field that is anchored in the deep interior, and if its poorly conducting surface layer is differentially rotating relative to the interior, the current generated by pulling the field line in the surface wind layer returns through the interior, producing Ohmic heating in the interior 4

As established above, the relevant part of heating for stalling the planet's contraction is the part that is deposited below $z_{\mathrm{tr}}$. The general criterion for stalling heating then translates to

$$
\left.\left.\int_{R}^{z_{\mathrm{tr}}} \frac{d Q}{d t}\right|_{\mathrm{Ohmic}} d z \approx F_{\text {rad }}\right|_{z_{\mathrm{tr}}} \approx \frac{\sigma_{B} T_{\mathrm{eq}}^{4}}{\tau_{\mathrm{tr}}}
$$

We express the planet's equilibrium temperature as $\sigma_{B}$ $T_{\mathrm{eq}}^{4} \sim L_{*} / 16 \pi a^{2}$ with $L_{*}$ the stellar luminosity and $a$ the stellar-planet separation. If an abnormal opacity source is present and causes a strong greenhouse effect, the value of $T_{\text {eq }}$ will be higher than adopted here.

The volumetric rate for Ohmic heating $d Q / d t=J^{2} / \sigma$, where $J$ is the electric current density and $\sigma$ is the electric conductivity. There are two characteristics of the profiles of Ohmic heating that pertain to this discussion, both demonstrated in Appendix A via an analytical model. First, as wind in the shallow layer pulls the poloidal magnetic field forward, a perturbed toroidal magnetic field, $b_{\phi}$, and a largely meridional current, $\mathbf{J} \approx J_{\theta} \mathbf{e}_{\theta}$, are created. Since the current is divergence-free, by geometry the radial current $J_{r}$ in this thin layer is roughly $\delta=z_{\text {wind }} / R \ll 1$ smaller than the meridional current, where $z_{\text {wind }}$ is the depth of the wind layer (eq. A7). The radial current flows continuously across the bottom of the wind zone, generating an interior radial current $J_{r}$ of a comparable magnitude (eq. A8). The interior $J_{\theta}$, however, is comparable to the local $J_{r}$ in this geometrically thicker zone (eq. A10). As a result the current density drops at the interface from $J \approx J_{\theta} \sim J_{r} R / z_{\text {wind }}$ above it to $J_{r}$ below. The local heating rate, $J^{2} / \sigma$, drops by a large factor of order $\left(R / z_{\text {wind }}\right)^{2}$ across the bottom of the wind zone. The bulk of the integrated Ohmic heating is deposited inside the wind layer, with only a fraction of order $\left(z_{\text {wind }} / R\right)$ deposited below it (Fig. 3). Our wind layer has a constant wind speed, but this discussion applies when, e.g. the wind speed varies sinusoidally with

4 We ignore current leakage from the top of the atmosphere, important for very hot atmospheres that sustain a significant ionization fraction (Perna et al. 2010b). 
depth as in Batygin \& Stevenson (2010), or decreases linearly with logarithmic pressure.

Second, since the interior current density $J \approx J_{r}$ is roughly constant below the wind layer, the interior volumetric heating rate decays as $1 / \sigma$. Since $\sigma$ rises rapidly with depth, most of the interior heating occurs right below the wind zone.

Following Batygin \& Stevenson (2010), we adopt a heating efficiency parameter $\epsilon$ that relates the total Ohmic heating (dominated by that in the wind layer) to the stellar insolation impinging on the planet,

$$
\int_{R}^{0} \frac{d Q}{d t} d z \approx \frac{J_{\text {wind }}^{2}}{\sigma_{\text {wind }}} z_{\text {wind }} \approx \epsilon \frac{L_{*}}{16 \pi a^{2}},
$$

where $J_{\text {wind }}$ and $\sigma_{\text {wind }}$ are evaluated in the wind layer. Now consider the part of Ohmic heating that occurs below $z_{\mathrm{tr}}$. We express the current there as $J_{\mathrm{tr}} \approx$ $J_{\text {wind }}\left(z_{\text {wind }} / R\right)$ and find

$$
\int_{R}^{z_{\mathrm{tr}}} \frac{d Q}{d t} d z \approx \frac{J_{\mathrm{tr}}^{2}}{\sigma_{\mathrm{tr}}} z_{\mathrm{tr}} \sim \epsilon \frac{L_{*}}{16 \pi a^{2}}\left(\frac{z_{\mathrm{wind}}}{R}\right)^{2} \frac{z_{\mathrm{tr}}}{z_{\mathrm{wind}}}\left(\frac{\sigma_{\mathrm{wind}}}{\sigma_{\mathrm{tr}}}\right) .
$$

Combining eq. (2) and the definition for $T_{\text {eq }}$, we obtain a constraint on $\epsilon$ if Ohmic heating is to be capable of stalling contraction,

$$
\epsilon \geq \frac{1}{\tau_{\mathrm{tr}}}\left(\frac{R}{z_{\mathrm{wind}}}\right)^{2} \frac{z_{\mathrm{wind}}}{z_{\mathrm{tr}}}\left(\frac{\sigma_{\mathrm{tr}}}{\sigma_{\mathrm{wind}}}\right)
$$

A larger $\epsilon$ is required to sustain a larger (higher entropy) planet, primarily because a higher entropy planet means a shallower $z_{\mathrm{tr}}$ and a markedly smaller $\tau_{\mathrm{tr}}$. If the wind penetrates only to a shallower depth $z_{\text {wind }}$, a higher $\epsilon$ is required to sustain the interior cooling. Note that this expression does not contain explicit dependence on the distance to the star. However, the conductivity in the wind zone $\left(\sigma_{\text {wind }}\right)$ drops with a smaller $T_{\text {eq }}$, in which case it becomes harder to sustain the cooling using Ohmic heating.

\subsection{Numerical Results}

To study the contraction of a planet under Ohmic heating, we construct a sequence of planet models that are in hydrodynamical and thermal equilibrium. We include the effects of stellar insolation and Ohmic heating; Ohmic heating is calculated self-consistently with an assumed efficiency $\epsilon$ and wind depth $z_{\text {wind }}$. In this section, we are interested in how Ohmic heating stalls the cooling contraction. So planets are assumed to be losing entropy and contracting in time. In $\$ 3$, we adopt the MESA code (Paxton et al. 2011) to study the problem of re-inflating planets after they have contracted.

A few details on the model building. We interpolate the tables of equation of state (Saumon et al. 1995) and opacity (Alexander \& Ferguson 1994 Ferguson et al. 2005. Cassisi et al. 2007) relevant for planetary mass objects. The center boundary condition is a dense solid core of size $100 \mathrm{~km}$, a density of $13 \mathrm{~g} / \mathrm{cm}^{3}$ and hence a small core mass of $\sim 5 \times 10^{22} \mathrm{~g}$. The outer boundary condition is that at the photosphere $p=(2 / 3) \mathrm{g} / \kappa$ and $T=T_{\text {eq }}=\left(L_{*} / 16 \pi \sigma_{B} a^{2}\right)^{1 / 4}$. We calculate the electron fraction for a solar composition gas (Anders \& Grevesse

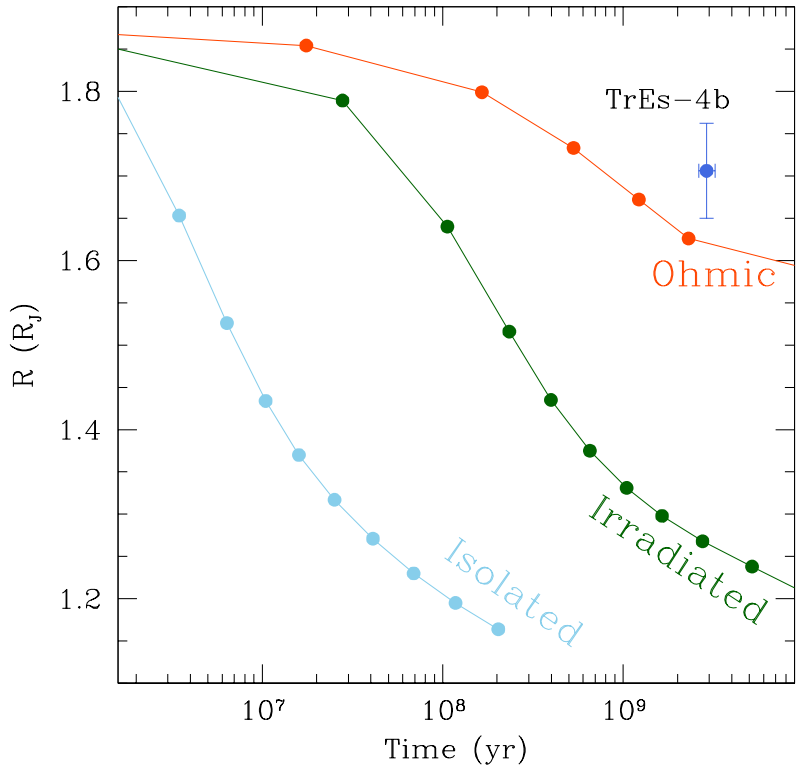

Fig. 2.- Cooling curves for TrEs4-b if it is in isolation (lowest curve), irradiated (middle curve) or receives both irradiation and Ohmic heating (upper curve). We adopt $\epsilon=3 \%$ and $z_{\text {wind }}=$ $2.5 \times 10^{8} \mathrm{~cm}$. The Ohmic mechanism stalls the cooling by replacing most of the cooling luminosity at the top of the convection zone with ohmic heating. The data point indicates the measurement for TrEs4-b.

1989), taking into account the thermal and pressure ionization of hydrogen, helium and three metals ( $\mathrm{Na}, \mathrm{K}$ and $\mathrm{Al}$, these are the elements with the lowest first ionization potential as well as high cosmic abundances). Metal contributions dominate near the surface while hydrogen is important in deeper regions. Electron conductivity is calculated using the electron-neutral collision cross-section from Draine et al. (1983). The depth of the wind layer is typically set to be $z_{\text {wind }}=2.5 \times 10^{8} \mathrm{~cm}$ (near pressure $p \sim 10$ bar) $~_{5}$ the surface dipole field strength is 5 Gauss. The wind velocity is adjusted so that the total Ohmic heating efficiency $\epsilon$ reaches the preset value, typically $3 \%$. The electric current is solved as a boundary value problem (see Appendix A), with $J_{r}=0$ at the surface and the electric potential $\Phi \propto r^{2}$ near the center. Heat is carried out by radiative diffusion, or convection where the temperature gradient is super-adiabatic. The planet model is iterated until the profile of Ohmic heating and the thermal structure are mutually compatible.

For each model, we must assume an intrinsic cooling luminosity $L_{\text {cool }}$. This is the net energy that is lost from the planet interior. The planet's internal entropy, as well as its size, are related to the total luminosity flowing through $z_{\text {tr }}$. We align models with different values of internal entropy into a time sequence, using the relation of $\int T \frac{d S}{d t} d^{3} x=L_{\text {cool }}$. The Ohmic heating does not contribute to the right-hand-side as it is an external energy source that hardly affects the bulk entropy of the planet.

5 In Appendix B we address to what extent the Lorentz force can accelerate fluid below the wind layer. We show that the resulting fluid velocities are negligible, and hence the wind profile adopted here is self-consistent. 


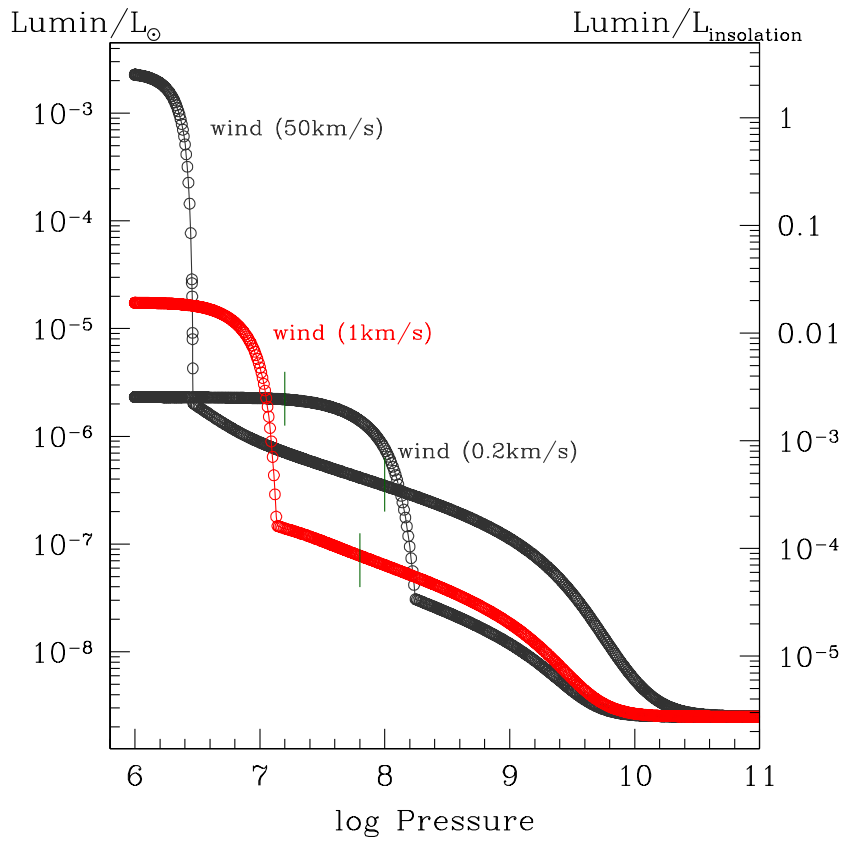

FIG. 3. - The radial profile of Ohmic heating, plotted as integrated luminosity (relative to solar luminosity on the left axis, and relative to insolation luminosity on the right axis) against logarithmic pressure (in cgs unit), using parameters similar to TrEs4$\mathrm{b}$, and when the planet has a radius of $1.6 R_{J}$. The features in the profile, including the jump at $z_{\text {wind }}$, are explained by our toy model in Appendix A The middle curve is our standard case with $z_{\text {wind }}=2.5 \times 10^{8} \mathrm{~cm}$ (at a pressure of $\sim 10 \mathrm{bar}=10^{7} \mathrm{dyne} / \mathrm{cm}^{2}$ ), while the other two are $z_{\text {wind }}=8 \times 10^{7} \mathrm{~cm}(\sim 3 \mathrm{bar})$, and $5 \times 10^{8} \mathrm{~cm}$ ( $\sim 100$ bar $)$, respectively. The large size of the planet requires a high internal entropy and a high luminosity at the top of the convection zone, marked here by the short vertical bars. This luminosity can be largely supplied by Ohmic heating if the wind is sufficiently deep and if the efficiency is sufficiently high (eq. (5)). The required Ohmic efficiency is $3 \%$ for our standard case, and $0.3 \%$ and $200 \%$ for the deeper and shallower cases, respectively.

We have also compared the results from our equilibrium models with those using MESA which integrates the temporal thermal evolution. We confirm that when we use the same Ohmic heating profile in MESA as in our equilibrium models, we obtain very similar results.

Fig. 2 shows results for the planet TrEs4-b. The cooling curves in three different scenarios are plotted. If TrEs4-b was born and evolved in isolation, it would have cooled to its current measured radii $\left(1.7 R_{J}\right)$ in less than $10 \mathrm{Myr}$; being irradiated by its host star at its current orbit raises this figure to $\sim 10^{8} \mathrm{yr}$; and having Ohmic heating on top of the irradiation prolongs it further to $\sim 10^{9}$ yr. Ohmic heating can be effective in stalling contraction.

The radial profile of Ohmic heating is displayed in Fig. 3. when TrEs-4b is at $1.6 R_{J}$. Less than a percent of the total Ohmic heating is deposited below $z_{\text {tr }}$ yet this amount is sufficient for suspending the contraction. Fig. 2 also demonstrates that when the wind layer is much shallower, the $\epsilon$ required is much larger (eq. 5).

Lastly, the radial profiles of electrical conductivity and gas temperature are displayed in Fig. 4. Compared to the models shown in Fig. 2 of B12, this planet model has a larger radius, a higher internal entropy, and a shallower $z_{\mathrm{tr}}$. In particular, the intense Ohmic heating near

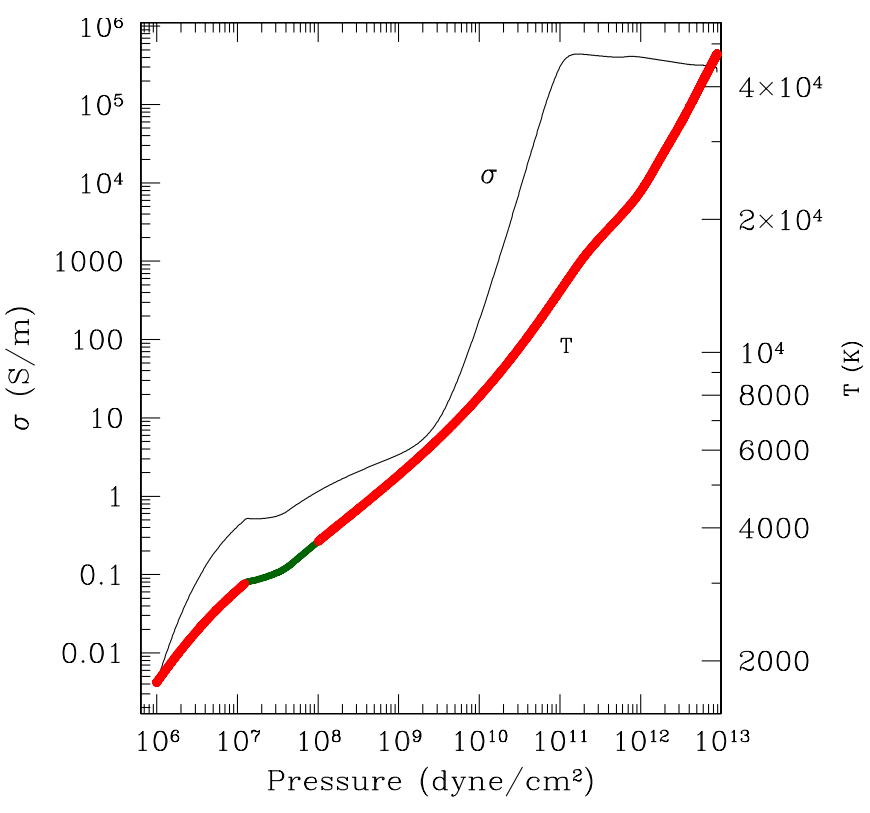

FIG. 4.- Electrical conductivity and gas temperature in a thermal equilibrium model for TrEs4-b, when subject to irradiation and Ohmic heating (according to the red $1 \mathrm{~km} / \mathrm{s}$ curve in Fig. 3). Near the surface, electrical conductivity is contributed by thermally ionized electrons from metals. Towards the interior, the contribution from hydrogen dominates. The temperature is shown in red when the region is convective and green when stably stratified. The entire wind zone is convective.

the surface causes the region above $\sim 10$ bar to become convective. A similar model without Ohmic heating will have an isothermal atmosphere (Fig. 11). The surface convection zone can significantly alter the local hydrodynamics.

The electrical conductivity we obtain differs from that shown in B12, but compares well against that in Huang \& Cumming (2012), a paper that appeared after the submission of this work. Given our assumption of constant total heating efficiency, the overall magnitude of $\sigma$ does not affect the results. But the radial profile (especially that near the surface) will. This may partly explain (see $\$ 4$ the difference between results here and those in B12

We proceed to calculate the expected radii at $1 \mathrm{Gyr}$ for the list of transiting planets, as compiled by Southworth (2011) (see http://www.astro.keele.ac.uk/jkt/tepcat), who has updated some of the values from previous publications through his homogeneous analysis. These are shown in Fig. 5 for which we assume that both insolation and Ohmic heating have acted on each planet since birth. We find that the expected radius excess can be roughly fitted by the following expression

$$
\frac{R-R_{J}}{R_{J}}=\frac{\Delta R}{R_{J}} \approx 0.5\left(\frac{T_{\mathrm{eq}}}{1500 \mathrm{~K}}\right)^{1.2},
$$

for planets at $\sim 1 M_{J}$. This results from the increasing conductivity and the larger irradiation luminosity when $T_{\text {eq }}$ is raised. This scaling is obtained for a constant heating efficiency of $3 \%$ (see $\$ 4$ for discussions). This scaling is compatible with the $\Delta R \propto T_{\mathrm{eq}}^{1.4 \pm 0.6}$ scaling obtained from the observed sample by Laughlin et al. (2011). For different planet masses, we find the excess decreases with mass: $T_{\mathrm{eq}} \approx 1500 \mathrm{~K}$, the excess scales 


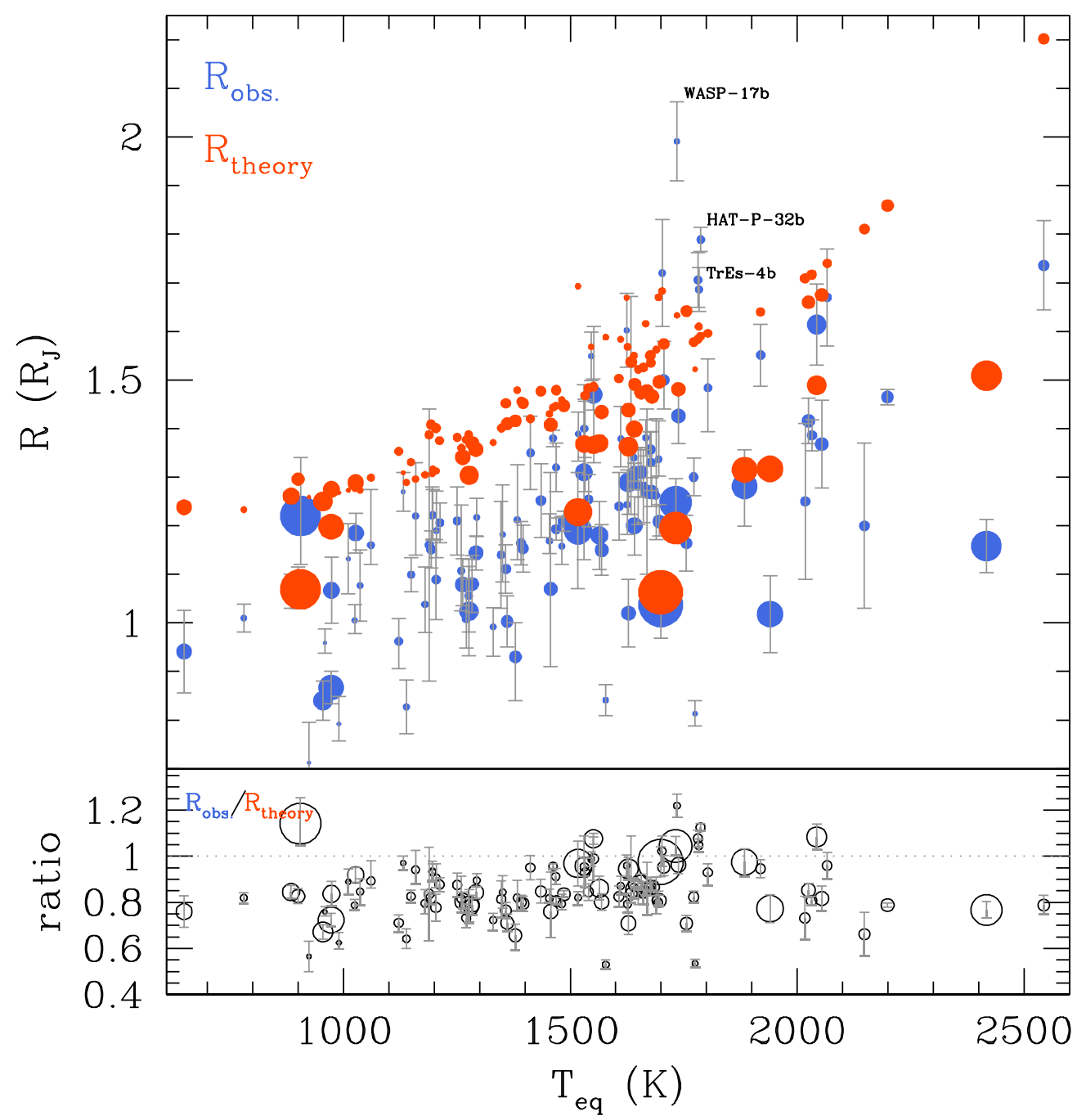

FIG. 5. - Expected radii at 1 Gyr of age for the list of transiting planets as compiled by Southworth (2011), plotted against their black-body equilibrium temperature. The sizes of the symbols correspond to planet masses (ranging between $\frac{2.2 M_{J}}{2}$ and $30 M_{J}$ ). We have adopted $\epsilon=3 \%$ and $z_{\text {wind }}=2.5 \times 10^{8} \mathrm{~cm}$. Our predicted radius excess, $\Delta R=R-R_{J} \propto T_{\mathrm{eq}}^{1.2}$ for $1 M_{J}$ planet, and $\Delta R \propto 1 / \sqrt{M}$ for planets with $T_{\mathrm{eq}} \approx 1500 \mathrm{~K}$. In contrast to B12, our calculations of Ohmic heating does not lead to runaway expansion, even for low mass planets. The lower panel plots the ratio between the observed and theoretical radii. Most planets have radii below the theoretical expectations, with the exception of WASP-17b and HAT-P-32b that have measured radii more than $2 \sigma$ above the theoretical values.

approximately as $\left(R-R_{J}\right) / R_{J} \sim 0.5\left(M / M_{J}\right)^{-1 / 2}$. More massive planets have smaller pressure scale heights for the same entropy.

The theoretical radii exceed those of almost all known hot jupiters, with the exceptions of a handful of planets at $T_{\mathrm{eq}} \sim 1700 \mathrm{~K}$. Among these, WASP-17b Anderson et al. 2011) and HAT-P-32b (Hartman et al. 2011) are larger than the theoretical values by more than $2 \sigma$. In fact, these two planets are so large their interiors are almost fully adiabatic. For planets with such a high entropy, $z_{\text {tr }}$ is almost at the photosphere, and hence an efficiency of Ohmic heating $\epsilon \geq 1$ (eq. 5) is required to stop the loss of entropy.

\section{CAN A COLD PLANET BE RE-INFLATED?}

We turn to consider the scenario where the planet has contracted significantly before it is subjected to intense stellar irradiation and Ohmic heating. This is possible in a variety of migration theories (e.g. Wu \& Murray 2003 Fabrycky \& Tremaine 2007; Wu \& Lithwick 2011;
Nagasawa et al. 2008) where the hot jupiters arrive at their current locations a few million to a few billion years after their formation.

We focus on WASP-17b $\left(0.49 M_{J}, T_{\text {eq }}=1770 \mathrm{~K}, \mathrm{An}-\right.$ derson et al. 2010, 2011), one of the lowest mass hot jupiters that is also heavily irradiated. These two factors should combine to produce the most pronounced reinflation $\sqrt{6}$ Since this is a non-equilibrium phenomenon, we employ the MESA package (Paxton et al. 2011), which has the option of a user-supplied routine for additional heating sources. The heating source we adopt has an energy generation rate per unit mass that scales quadratically with pressure above $z_{\text {wind }}$, dropping by a factor of $\left(R / z_{\text {wind }}\right)^{2}$ at $z_{\text {wind }}$, and scales as $p^{-1.3}$ below the wind zone until $p=10^{10}$ dyne $/ \mathrm{cm}^{2}$, below which the high conductivity leads to a diminishingly small heat-

6 We note that the observed radius of WASP-17b is too big to explain even if Ohmic heating commences at formation. We use its extreme properties to illustrate the inefficacy of Ohmic re-inflation. 


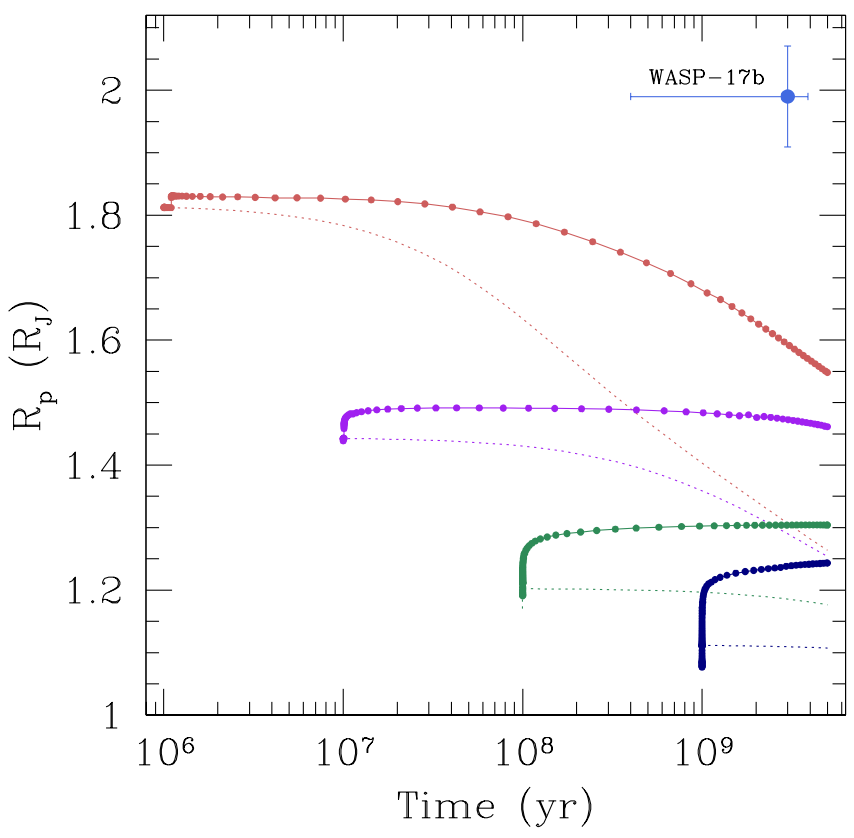

FIG. 6. - The re-inflation of WASP-17b (the data point at the top right) by Ohmic heating $(\epsilon=3 \%)$ and stellar insolation $\left(T_{\mathrm{eq}}=\right.$ $1770 \mathrm{~K}$ ). Different tracks of radius evolution (solid curves with points) correspond to initial models that have cooled in isolation for $10^{6}, 10^{7}, 10^{8}$ and $10^{9} \mathrm{yrs}$, respectively (from top to bottom) Once the core entropy has been reduced to a low level, heating in the atmosphere only has a limited effect on the radius. The dotted curves show the same evolutionary models with stellar irradiation only.

ing that scales as $p^{-4}$. Such a profile (the integrated heating is shown in Fig. 3) closely approximates the actual Ohmic heating profile we calculate for WASP-17b and agrees with our analytical model in Appendix A. The results on radius expansion are shown in Fig. 6 where planet models are exposed to both Ohmic heating (3\%) and surface irradiation (with $T=T_{\text {eq }}$ ), after having cooled in isolation for a period ranging from $10^{6}$ to $10^{9}$ yrs.

The MESA calculations show that re-inflation is ineffective: the planet can at best be re-inflated by $\sim 10 \%$ if it has already cooled (Fig. 6). In Fig. 7, we illustrate in detail why this is so. Ohmic heating is intrinsically shallow and most of the energy is deposited at $p<10 \mathrm{bar}=10^{7} \mathrm{dyn} / \mathrm{cm}^{2}$, whereupon most of it is quickly transported outward by the newly generated surface convection zone. The interior of the planet experiences the heating only when a temperature inversion is built up and heat diffuses gradually inward ${ }^{7}$ By the time heat has diffused downward to $p=10^{11}$ dyne $/ \mathrm{cm}^{2}$, the temperature inversion is minimal and the radiative diffusion has nearly ground to a halt. As a result, only layers shallower than this experience appreciable heating. Since the pressure scale height is $\sim c_{s}^{2} / g \sim$ $4 \% R_{J}(T / 7000 \mathrm{~K})^{1 / 2}$, the planet can at most expand a few times this value. This result has significance for the survival of hot jupiters.

${ }^{7}$ It is often claimed that as long as heat is deposited somewhere in the convection zone, it will be mixed uniformly. This is not true. Heat can not be transported against the temperature gradient, except with the help of external forcing.

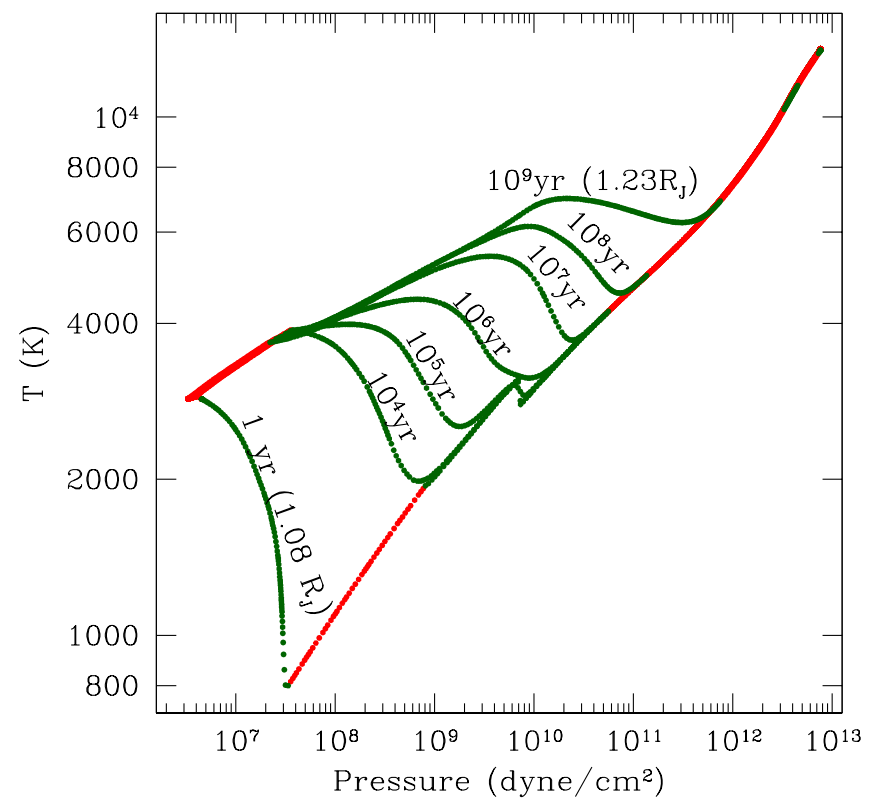

FIG. 7.- Evolution of the temperature structure in a WASP-17b like planet, under Ohmic heating $(3 \%)$ and stellar irradiation, after the planet has cooled in isolation for 1 Gyrs (the lowest trajectory in Fig. 6. The outer most point lies at an optical depth of 100. The individual curves are snapshots taken at different epochs after the heating has commenced. The planet is eventually mildly reinflated to $1.25 R_{J}$ after 5 Gyrs. With time, a thicker and thicker surface layer of the planet is lifted from the original adiabat to a higher adiabat. Ohmic heating below $p \approx 10^{7}$ dyne $/ \mathrm{cm}^{2}$ is typically so small that it does not contribute much to the temperature rise there. Instead, heat diffuses downward gradually with the help of a temperature inversion. Red color indicates convective regions and green radiative regions. The wind zone is convective.

In contrast to our result here, $\mathrm{B} 12$ find that low mass planets can be reinflated by Ohmic heating to become Roche Lobe overflowing. In fact, B12 typically find a more drastic effect of Ohmic heating than that obtained here: our TrEs-4b model reaches an equilibrium radius of $1.6 R_{J}$ (Fig. 2), while a similar model in B12 yields $1.9 R_{J}$ (Fig. 5 in B12). Detailed differences in, e.g., radial profiles of conductivity and wind, treatment of radiative atmosphere, between our models may go some way in explaining this difference. However, re-inflation by of order unity requires raising temperature in the planetary center (bottom scale height). We find that radiative diffusion could not accomplish this (Fig. 7).

Fig. 7 also demonstrates that heating a cold planet from the outside produces a much more pronounced radiative region (compare with Fig. 4). This may have consequences for tidal dissipation, elemental diffusion and magnetic dynamo in the planet.

In conclusion, we find that it is difficult to re-inflate by a substantial amount a planet that has contracted.

\section{DISCUSSION ON UNCERTAINTIES}

We clarify two issues here. Our assumption of a fixed Ohmic efficiency is ad hoc. It allows us to focus on the thermodynamics of Ohmic heating, but obscures the atmospheric physics that determines the actual wind speed, as well as any dependence on planet temperature or magnetic field strength. Menou (2011) and B12 have argued that, in the weather layer $\sim 0.1 b a r)$ where stellar inso- 
lation produces a large horizontal pressure gradient, the wind is driven by the thermal gradient but dragged by the Lorentz force. Assuming the two forces balance each other, Menou (2011) obtains an equilibrium wind speed and an Ohmic heating efficiency (in the weather layer) that peaks at a few percent when the surface temperature is $\approx 1500 \mathrm{~K}(B \sim 10 \mathrm{G})$. However, this argument may be incomplete. The biggest Lorentz drag is applied not at the weather layer, but deeper down, at the bottom of the wind zone where conductivity is the highest (see, e.g. Liu et al. 2008). So the actual wind speed depends on how deep the wind zone extends to. This in turns depends on how well the weather layer couples dynamically to the deeper atmosphere, a process currently not well understood (Perna et al. 2010a; Rauscher \& Menou 2010: Lian \& Showman 2008; Liu \& Schneider 2010). An added complication is that Ohmic heating itself can modify the local temperature gradient and stratification (see below). Further study is necessary.

An additional question is whether heat deposited above $z_{\text {tr }}$ can affect the radius evolution. This is the majority of Ohmic heating and can be characterized by GCM simulations. We argue (\$2 that this fraction of heat cannot directly supplant the internal entropy loss and halt the contraction. But it raises the local temperature gradient (Fig. 4 ${ }^{8}$ and exerts an indirect effect on the radius evolution, as is observed in our numerical results. At a given interior adiabat, an atmosphere with such a superficial heating has a deeper $z_{\mathrm{tr}}$ than an atmosphere that is only irradiated and isothermal. Deeper $z_{\text {tr }}$ implies that the amount of energy necessary to suspend internal cooling, the one that is deposited below $z_{\mathrm{tr}}$ ), is reduced (see $\$ 2$ ). The rising temperature in the wind zone brings about a greater conductivity, a larger magnetic drag, and likely, a smaller wind speed. This could lead to saturation of Ohmic heating.

\section{CONCLUSION}

We study the radius evolution of hot jupiters under Ohmic heating and stellar insolation. We consider initial conditions in which either (a) the planet starts with an high entropy, or (b) it has previously cooled and contracted. In the former case, we find that planets can be sustained at large radii for billions of years (Fig. 5). For our example case of TrEs-4b, the planet may remain in a state of perpetual youth, at a cooling age of $10 \mathrm{Myr}$ (Fig. 2). Less massive planets can be suspended at larger radii than the more massive ones in the same radiation environment. We place a constraint on the Ohmic heating if it is to suspend the cooling contraction: for an efficiency of a few percent, the wind layer needs to extend to a depth of order 10 bar; a shallower layer would require a higher heating efficiency (eq. 5).

We also consider Ohmic heating on planet models that have previously contracted. Ohmic heating, being very superficial, has a limited capacity for re-inflating these planets. We illustrate this using WASP-17b, a low mass planet that has one of the highest irradiation fluxes, yet still cannot be significantly inflated after cooling (Fig. 6). As such, Ohmic heating is best termed 'Ohmic suspension', rather than 'Ohmic inflation'.

The interior structure of the planet is affected by Ohmic heating: the planet has an adiabatic interior and a largely isothermal atmosphere in the case of no Ohmic heating, but the wind zone tends to become convective when Ohmic heating is applied. This may impact on the physics of global circulation, magnetic dynamo, tidal dissipation and element settling.

While a few planets have observed radii comparable to or even larger than predictions using our fiducial Ohmic heating parameters (WASP-17b and HAT-P-32b, in particular), the vast majority fall below these predictions. In fact, many are consistent with cooling without any Ohmic heating, and some are too small for pure gaseous spheres. There could be a number of explanations for these. They could have lower Ohmic heating efficiencies than stipulated here, or they could have massive solid cores and/or heavy-metal enriched envelopes (see, e.g. Baraffe et al. 2008), or, alternatively, they may have a different migration history, as we now describe. Consider the scenario where the planet is migrated to its current location well after formation. Before the migration, it contracts and loses entropy in isolation. If during the migration there is no significant entropy injection into the deep interior of the planet, the planet will then follow one of the trajectories in Fig. 6 and end up having a different radius depending on its time of migration. If this scenario is correct, it would mean that the present day planet radii retain the memory of their past dynamical history.

There is a caveat to our above scenario. During the migration process, there is likely a large amount of tidal energy deposited inside the planet and this may have rejuvenated the planet. However, there are substantial theoretical uncertainties regarding the mechanism and location of tidal heating. We hope to address tidal rejuvenation in an upcoming publication.

The super-sizes of WASP-17b and HAT-P-32b are surprising: if Ohmic heating is responsible for their sizes, it must be both very efficient and remarkably continuous. For instance, if the dynamo field weakens for, say, $10^{8}$ years, the internal entropy would be mostly drained away and the planet would have contracted.

We thank the referee, Kristen Menou, for suggestions that improved the clarity of this paper. Y.W acknowledges useful conversations with P. Goldreich, T. Guillot, K. Batygin, P. Arras, and especially thanks B. Paxton and other MESA creators for this wonderful package. Y.L. acknowledges support from NSF grant AST1109776 .

\section{REFERENCES}

Alexander, D. R. \& Ferguson, J. W. 1994, ApJ, 437, 879

Anders, E. \& Grevesse, N. 1989, Geochim. Cosmochim. Acta, 53, 197

\footnotetext{
${ }^{8}$ In more extreme cases (Fig. 7), temperature gradient near the surface is even inverted.
}

Anderson, D. R., Hellier, C., Gillon, M., Triaud, A. H. M. J., Smalley, B., Hebb, L., Collier Cameron, A., Maxted, P. F. L., Queloz, D., West, R. G., Bentley, S. J., Enoch, B., Horne, K., Lister, T. A., Mayor, M., Parley, N. R., Pepe, F., Pollacco, D., Ségransan, D., Udry, S., \& Wilson, D. M. 2010, ApJ, 709, 159 
Anderson, D. R., Smith, A. M. S., Lanotte, A. A., Barman, T. S., Collier Cameron, A., Campo, C. J., Gillon, M., Harrington, J., Hellier, C., Maxted, P. F. L., Queloz, D., Triaud, A. H. M. J., \& Wheatley, P. J. 2011, MNRAS, 416, 2108

Arras, P. \& Bildsten, L. 2006, ApJ, 650, 394

Baraffe, I., Chabrier, G., \& Barman, T. 2008, A\&A, 482, 315

Batygin, K. \& Stevenson, D. J. 2010, ApJ, 714, L238

Batygin, K., Stevenson, D. J., \& Bodenheimer, P. H. 2011, ApJ, 738,1

Bodenheimer, P., Lin, D. N. C., \& Mardling, R. A. 2001, ApJ, 548, 466

Burrows, A., Hubeny, I., Budaj, J., \& Hubbard, W. B. 2007, ApJ, 661, 502

Cassisi, S., Potekhin, A. Y., Pietrinferni, A., Catelan, M., \& Salaris, M. 2007, ApJ, 661, 1094

Chabrier, G. \& Baraffe, I. 2007, ApJ, 661, L81

Charbonneau, D., Brown, T. M., Latham, D. W., \& Mayor, M. 2000, ApJ, 529, L45

Draine, B. T., Roberge, W. G., \& Dalgarno, A. 1983, ApJ, 264, 485

Enoch, B., Cameron, A. C., Anderson, D. R., Lister, T. A., Hellier, C., Maxted, P. F. L., Queloz, D., Smalley, B., Triaud, A. H. M. J., West, R. G., Brown, D. J. A., Gillon, M., Hebb, L., Lendl, M., Parley, N., Pepe, F., Pollacco, D., Segransan, D., Simpson, E., Street, R. A., \& Udry, S. 2011, MNRAS, 410, 1631

Fabrycky, D. \& Tremaine, S. 2007, ApJ, 669, 1298

Ferguson, J. W., Alexander, D. R., Allard, F., Barman, T., Bodnarik, J. G., Hauschildt, P. H., Heffner-Wong, A., \& Tamanai, A. 2005, ApJ, 623, 585
Hartman, J. D., Bakos, G. Á., Torres, G., Latham, D. W., Kovács, G., Béky, B., Quinn, S. N., Mazeh, T., Shporer, A., Marcy, G. W., Howard, A. W., Fischer, D. A., Johnson, J. A., Esquerdo, G. A., Noyes, R. W., Sasselov, D. D., Stefanik, R. P., Fernandez, J. M., Szklenár, T., Lázár, J., Papp, I., \& Sári, P. 2011, ApJ, 742, 59

Huang, X. \& Cumming, A. 2012, ApJ, 757, 47

Laughlin, G., Crismani, M., \& Adams, F. C. 2011, ApJ, 729, L7

Lian, Y. \& Showman, A. P. 2008, Icarus, 194, 597

Liu, J., Goldreich, P. M., \& Stevenson, D. J. 2008, Icarus, 196, 653

Liu, J. \& Schneider, T. 2010, Journal of Atmospheric Sciences, 67,3652

Menou, K. 2011, ArXiv e-prints

Nagasawa, M., Ida, S., \& Bessho, T. 2008, ApJ, 678, 498

Paxton, B., Bildsten, L., Dotter, A., Herwig, F., Lesaffre, P., \& Timmes, F. 2011, ApJS, 192, 3

Perna, R., Heng, K., \& Pont, F. 2012, ApJ, 751, 59

Perna, R., Menou, K., \& Rauscher, E. 2010a, ApJ, 719, 1421

-. 2010b, ApJ, 724, 313

Rauscher, E. \& Menou, K. 2010, ApJ, 714, 1334

Saumon, D., Chabrier, G., \& van Horn, H. M. 1995, ApJS, 99, 713

Showman, A. P., Cho, J. Y.-K., \& Menou, K. Atmospheric

Circulation of Exoplanets, ed. Seager, S., 471-516

Southworth, J. 2011, MNRAS, 417, 2166

Wu, Y. \& Lithwick, Y. 2011, ApJ, 735, 109

Wu, Y. \& Murray, N. 2003, ApJ, 589, 605

Youdin, A. N. \& Mitchell, J. L. 2010, ApJ, 721, 1113

APPENDIX

\section{APPENDIX A: THE RADIAL PROFILE OF OHMIC HEATING}

Here we describe our model for Ohmic heating. We assume a fixed wind profile near the surface of the planet, and a dipolar magnetic field threading the planet. We ignore here the effect of the Lorentz force on the fluid velocity, but return to discuss it in Appendix B. The electric current is obtained by solving

$$
\begin{aligned}
\boldsymbol{J} & =\sigma\left(-\nabla \Phi+\frac{\mathbf{v}_{\text {wind }} \times \mathbf{B}_{\text {dipole }}}{c}\right) \\
\nabla \cdot \boldsymbol{J} & =0,
\end{aligned}
$$

subject to the boundary conditions that $J_{r}=0$ at the planet's surface $(r=R)$ and center $(r=0)$. We have used that the steady state electric field is the gradient of a potential, $\boldsymbol{E}=-\boldsymbol{\nabla} \Phi$. The dipolar field is

$$
\boldsymbol{B}_{\text {dipole }}=-\nabla\left(\frac{\mathbf{M} \cdot \mathbf{r}}{r^{3}}\right)=M \frac{\left(2 \cos \theta \mathbf{e}_{r}+\sin \theta \mathbf{e}_{\theta}\right)}{r^{3}},
$$

in spherical co-ordinates, and we assume that the wind has a constant angular velocity $\omega$ in a zone that extends to a depth $\delta R$, i.e.

$$
\boldsymbol{v}_{\text {wind }}=\omega r \sin \theta \boldsymbol{e}_{\phi} \quad \text { for } 1-\delta<r / R<1
$$

and is zero for $r / R<1-\delta$. The above equations combine to yield

$$
\boldsymbol{J}=-\sigma \boldsymbol{\nabla}\left(\Phi+\frac{2 \omega M}{3 c r}\left(P_{2}-P_{0}\right)\right) \quad \text { for } 1-\delta<r / R<1
$$

and $\boldsymbol{J}=-\sigma \nabla \Phi$ below the wind zone. We discard the uninteresting term $P_{0}$ and assume all variables follow a $P_{2}$ dependence, adopting the form $\Phi=\Phi_{2}(r) P_{2}$ for the potential. In the body of the paper, we numerically solve the ODE for $\Phi_{2}$ that results from setting $\boldsymbol{\nabla} \cdot \boldsymbol{J}=0$ to obtain the Ohmic heating rate, which is the volume-integrated $J^{2} / \sigma$.

In the remainder of this Appendix, we solve analytically a simple example that illustrates two important features of Ohmic heating: first, the rapid drop in the heating profile by the factor $\sim \delta^{2}$ just below the wind, and second, the inward decay in the heating profile in the interior (see numerical results in Fig. 3). We take $\sigma$ to be a piecewise function of radius, with $\sigma=\sigma_{\text {shallow }}$ through the wind zone to a depth $\Delta R$, where $\Delta>\delta$; and $\sigma=\sigma_{\text {deep }}$ below that. There are then three shells of interest. In the outer one $(1-\delta<r / R<1)$, there is a wind and $\sigma=\sigma_{\text {shallow; }}$ in the middle one $(1-\Delta<r / R<1-\delta)$, there is no wind and $\sigma=\sigma_{\text {shallow }}$; and in the inner one $(r / R<1-\Delta)$ there is no wind and $\sigma=\sigma_{\text {deep. }}$. The ODE for $\Phi_{2}$ is easily solved analytically in each shell, and between the shells one requires 
that $\Phi$ and $J_{r}$ are continuous. Omitting the algebra, we find that in the limit $\delta<\Delta \ll 1$ and $\sigma_{\text {shallow }} \ll \sigma_{\text {deep }}$ the current in each of the three shells is given by

$$
\begin{array}{rc}
\text { Outer shell (wind zone) : } & J_{r} \approx-6\left(1-\frac{r}{R}\right) \cdot J_{0} P_{2}, \quad J_{\theta} \approx J_{0} d P_{2} / d \theta, \\
\text { Middle shell : } & J_{r} \approx-6 \delta \cdot J_{0} P_{2}, \quad J_{\theta} \approx 6 \delta\left(1-\frac{r}{R}-\Delta\right) \cdot J_{0} d P_{2} / d \theta, \\
\text { Inner shell : } & J_{r} \approx-6 \frac{r}{R} \delta \cdot J_{0} P_{2}, \quad J_{\theta} \approx-3 \frac{r}{R} \delta \cdot J_{0} d P_{2} / d \theta
\end{array}
$$

where the constant

$$
J_{0} \equiv \frac{2 \omega M \sigma_{\text {shallow }}}{3 c R^{2}}
$$

Hence the total current in the wind zone is dominated by $J_{\theta}$ which is $\sim 1 / \delta$ greater than the radial current $J_{r}$. Below the wind zone, $J_{r}$ is continuous while $J_{\theta}$ drops discontinuously to less than $J_{r}$. This causes a large drop in the rate of Ohmic heating $\left(J^{2} / \sigma\right)$ below the wind zone, of order $\delta^{2}=\left(z_{\text {wind }} / R\right)^{2}$. Moreover, the higher conductivity in the core ensures that there is little Ohmic dissipation there. Substituting values of $\delta=0.001$, and $\Delta=0.005$ and $\sigma_{\text {deep }} / \sigma_{\text {shallow }}=10^{7}$, the fractions of the total ohmic dissipation taking place in the outer, middle, and inner shells are $0.999855,0.000144851,9.04343 \times 10^{-10}$, respectively We argue in the text that only heating in the middle shell can contribute to halting the planet's contraction.

\section{APPENDIX B: DYNAMICAL EFFECTS BELOW THE WIND ZONE}

While wind is forced in the outer shell, there is no external forcing in the inner shells. A simple estimate for the Lorentz force $(\mathbf{J} \times \mathbf{B} / c)$ shows that the inner layers could be spun up in a short timescale. We investigate the dynamical response of the planet to the surface battery by looking for steady-state solution to the following equations,

$$
\begin{gathered}
\rho \frac{\partial \mathbf{v}}{\partial t}=\frac{\mathbf{J} \times \mathbf{B}}{c} \\
\frac{1}{c} \frac{\partial \mathbf{b}}{\partial t}=-\nabla \times \mathbf{E} .
\end{gathered}
$$

Here, $\mathbf{b}$ denotes perturbed field line, while $\mathbf{B}$ the background field. At steady state, $\mathbf{J}=\nabla \times \mathbf{b}$.

We will study a cylindrical analog to the planet where the cylindrical $z$ direction approximates that of the spherical $\theta$ direction, and cylindrical radius that of the spherical radius. The dipole field is now $\mathbf{B}=B_{r} \mathbf{e}_{\mathbf{r}}$ with $B_{r}=B_{0} R / r$.

We include only the components $\mathbf{b}=b_{\phi} \mathbf{e}_{\phi}$ and $\mathbf{j}=j_{z} \mathbf{e}_{\mathbf{z}}$. We further assume a harmonic $z$ dependence $\propto e^{i k_{z} z}$.

In the wind zone, an external forcing, $\mathbf{F}=F \mathbf{e}_{\phi}$ maintains the current. The time-independent equations are now,

$$
\begin{aligned}
\frac{B_{r}}{c} \frac{1}{r} \frac{\partial}{\partial r}\left(r b_{\phi}\right)+F & =0 \\
-\frac{k_{z}^{2}}{\sigma} b_{\phi}+\frac{\partial}{\partial r}\left(\frac{1}{\sigma r} \frac{\partial}{\partial r}\left(r b_{\phi}\right)\right)-\frac{B_{r}}{c} \frac{\partial v_{\phi}}{\partial r} & =0 .
\end{aligned}
$$

The boundary condition is that the radial current vanishes at $r=R$, or $b_{\phi}=0$ at $R$. Taking $r \approx R$, and a constant forcing $F$, we find the solution to be

$$
\begin{aligned}
& b_{\phi}=\frac{F c R}{B_{0}}(1-r / R) \\
& v_{\phi} \approx v_{\text {wind }}=\text { const. }
\end{aligned}
$$

since we take the wind zone be of thickness $\delta \ll 1 \sim 1 /\left(R k_{z}\right)$.

At the bottom of the wind zone, $b_{\phi}$ behaves continuously (radial current has to be continuous), but a jump in the tangential velocity $(\Delta v)$ is necessary to ensure that the tangential electric field is continuous. The velocity there is

$$
v_{\phi}(r=R-\delta)=v_{\text {wind }}-\Delta v=v_{\text {wind }}-\frac{F c^{2}}{B_{r}^{2} \sigma} \text {. }
$$

Further below, both the velocity and $b_{\phi}$ decay inward as,

$$
\begin{aligned}
b_{\phi} & =\frac{F c R \delta}{B_{0} r} e^{i k_{z} z} \\
\frac{\partial v_{\phi}}{\partial r} & =-\frac{F c^{2} k_{z}^{2} \delta}{B_{0}^{2} \sigma} e^{i k_{z} z} .
\end{aligned}
$$

So the region of lowest $\sigma$ is also where $v_{\phi}$ decreases most steeply. Compared to $\Delta v$, the total shear across the no-forcing zone is much smaller,

$$
v_{\phi}(r=R-\delta)-v_{\phi}(r=0) \sim k_{z}^{2} \delta^{2} \Delta v \ll \Delta v .
$$


This result indicates that, at steady state, the interior is able to maintain a largely uniform rotation with $v_{\phi} \approx v_{\phi}(r=$ $R-\delta)$. Contrary to our initial expectation, the interior can rotate at a different rate than that in the wind zone, despite the large Lorentz torque. It is not spun up.

A few words on the energetics. Stellar insolation drives the wind which suffers both turbulent dissipation and Ohmic loss. We take the the timescale for turbulent dissipation of the wind to be the wind travelling time across the planet, $R / v_{\text {wind }}$

$$
\left(\frac{d E}{d t}\right)_{\mathrm{turb}} \approx 2 \pi r \delta \frac{\rho v_{\mathrm{wind}}^{3}}{R}
$$

and the rate for Ohmic loss is

$$
\left(\frac{d E}{d t}\right)_{\mathrm{ohmic}} \approx 2 \pi r \delta F v_{\text {wind }}=2 \pi r \delta v_{\text {wind }}^{2} \frac{B_{r}^{2} \sigma}{c^{2}} .
$$

We find the ratio between the two rates to be

$$
\frac{(d E / d t)_{\text {turb }}}{(d E / d t)_{\text {ohmic }}} \approx \frac{\rho v_{\text {wind }} c^{2}}{R B_{r}^{2} \sigma} .
$$

Adopting parameters $\rho=10^{-4} \mathrm{~g} / \mathrm{cm}^{3}, \sigma=10^{10} \mathrm{~s}^{-1}, B_{r}=5 \mathrm{G}$ and $v_{\text {wind }}=10^{5} \mathrm{~cm} / \mathrm{s}$, this ratio is $\approx 4$, or Ohmic loss is non-negligible. This suggest that the Ohmic efficiency may be higher than the canonical $3 \%$ we adopt in the text. 